\title{
Surgery for ulcerative colitis in the era of the pouch: The St Mark's Hospital experience
}

\author{
D M Melville, J K Ritchie, R J Nicholls, P R Hawley
}

\begin{abstract}
The choice of operation for ulcerative colitis among 422 patients having all their surgery at one hospital between 1976 (the year of the first restorative proctocolectomy) and 1990, was reviewed. The 15 year period was divided into three quinquennia (1976-80, 1981-85, 1986-90). Elective surgery was performed in 316 patients with one operative death. The proportions of conventional proctocolectomy, colectomy with ileorectal anastomosis, and restorative proctocolectomy for the three quinquennia were $36 / 60,17 / 60,4 / 60$; 29/111, 30/111, 35/111; 30/145, 17/145, 75/145. Of 106 urgent operations with three postoperative deaths, 12 had a conventional proctocolectomy and 86 a colectomy with ileostomy and preservation of the rectum. Of 85 survivors of the latter there were two late deaths and in 13 no further surgery had been done at the time of this assessment. In the remaining 70 having subsequent surgery the proportion of conventional proctocolectomy, colectomy with ileorectal anastomosis, and restorative proctocolectomy for the three quinquennia respectively were $19 / 27,4 / 27,14 / 27 ; 11 / 21$, $2 / 21,8 / 21 ; 5 / 22,4 / 22,13 / 22$. Of the 76 patients having colectomy with ileorectal anastomosis $12(16 \%)$ no longer had a functioning rectum at the end of 1990 . Of the 153 patients having an ileoanal pouch procedure, $11(7 \%)$ no longer had, a functioning anus. The study showed an increase in the numbers of patients having elective surgery for ulcerative colitis during the three quinquennia. It also showed a rise of restorative over conventional proctocolectomy with diminution in elective colectomy with ileorectal anastomosis in the last five year period.

(Gut 1994; 35: 1076-1080)
\end{abstract}

In the 50 years since the first definitive surgery for ulcerative colitis was undertaken at this hospital, the choice of operations for patients requiring surgery has widened considerably. Total proctocolectomy as a single or staged procedure and colectomy and ileorectal anastomosis were the mainstays of treatment until 1976. In that year Sir Alan Parks carried out the first operation here entailing removal of all the large bowel with formation of a pelvic pouch as a single procedure; an operation known at this centre as restorative proctocolectomy. ${ }^{1}$ As a result greater efforts have also been made to preserve the anal sphincters in patients requiring proctectomy but not initially suitable for the formation of a pelvic pouch. Such an operation entailing excision of the colon and most of the rectum with formation of an end ileostomy is now usually termed conservative proctocolectomy. Even when the anal sphincters have been removed, a Kock intra-abdominal reservoir remains as a possibility. Such a range of operations makes the decision difficult in any given case, both for patient and surgeon.

This paper describes the changes in surgical practice in the treatment of ulcerative colitis over the 15 years since the introduction of the pelvic pouch. Changes in surgical practice have been analysed as have the successes and failures of the various procedures but longterm detailed functional outcome in pouch patients has not been included as these data are reported in this issue. ${ }^{2}$

\section{Patients and methods}

The series consists of all patients who had their first surgery for ulcerative colitis (as shown by the pathology report on the specimen) at this hospital from 1976-90. Nineteen patients in whom the specimen showed indeterminate or unclassified colitis have been included in these figures: none had shown evidence of Crohn's disease by the end of 1990. Patients in whom the first operation was undertaken elsewhere and were later referred for further surgery (usually for treatment of the retained rectum after a colectomy and ileostomy) have been excluded to avoid bias in the figures. The total number of patients (422) has been divided into those receiving elective surgery and patients operated on urgently. All patients have been allocated to one of these two groups by one author throughout using the basic classification put forward by Ewart and Lennard-Jones. ${ }^{3}$ Elective surgery includes groups $\mathrm{A}$ and $\mathrm{B}$ of this classification - that is, patients in good general condition with inactive or mildly active colitis. Patients in group $\mathrm{C}$ - that is, those in poor general condition or severe active colitis, or both, and group D - that is, those who are critically ill and undergo emergency operations have been amalgamated under the heading of urgent surgery.

\section{Results}

\section{ELECTIVE SURGERY}

Three hundred and sixteen patients were operated upon electively in the 15 year period
Accepted for publication 29 October 1993 


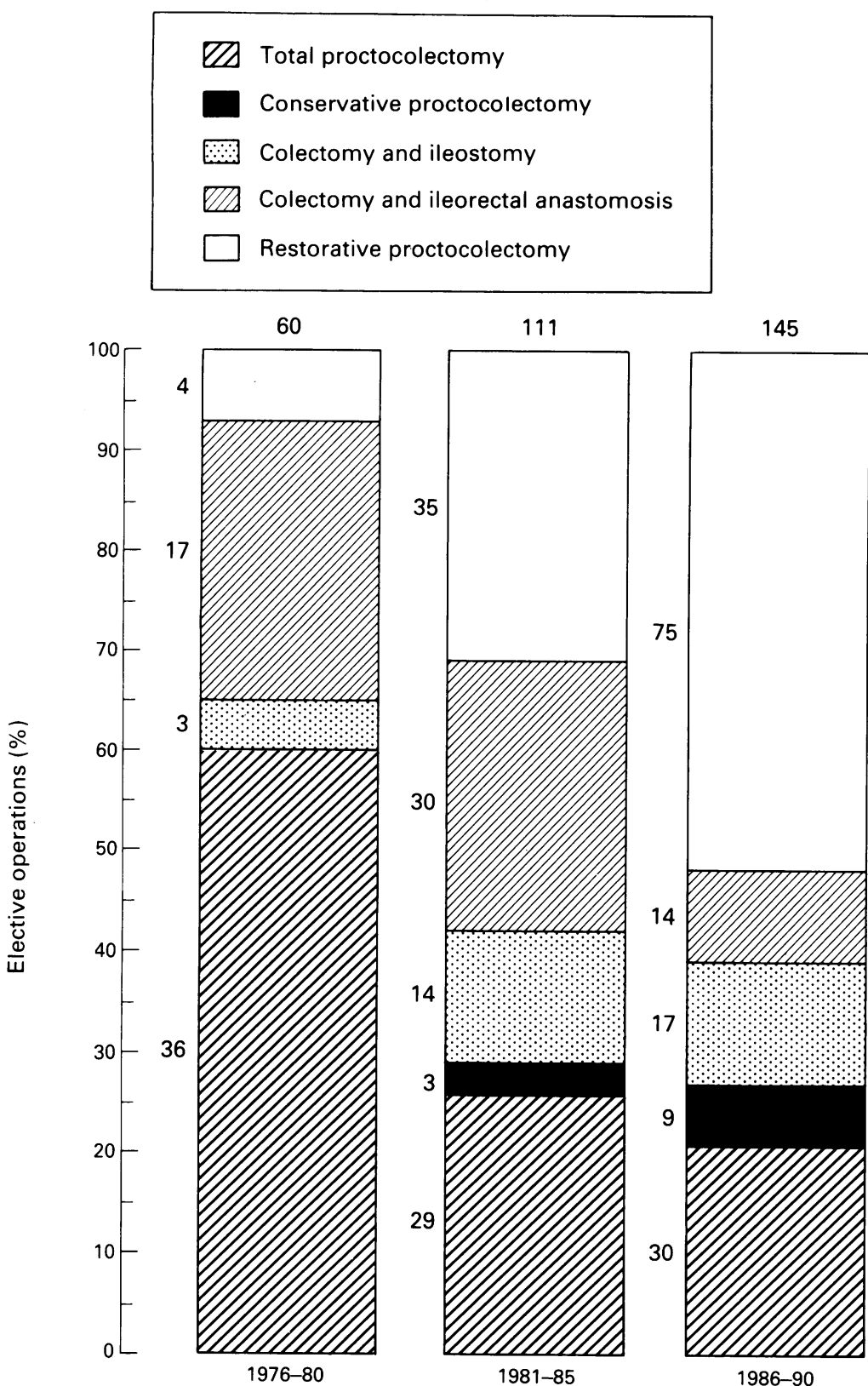

Figure 1: Elective operations performed 1976-90.

and the operations were total proctocolectomy 95 , conservative proctocolectomy 12 , colectomy and ileostomy 34, colectomy and ileorectal anastomosis 61 , and restorative proctocolectomy 114. There was one postoperative death. This 81 year old man with underlying idiopathic thrombocytopenic purpura, died 11 days postoperatively after a total proctocolectomy for carcinoma of the rectum in ulcerative colitis. It was impossible to withdraw ventilatory support at any stage postoperatively. Figure 1 shows the figures divided into three quinquennia: 1976-80, 1981-85, and 1986-90. The numerical and percentage increase in patients having restorative proctocolectomy is accounted for in large part by patients referred to the hospital specifically for this procedure. The percentage of patients treated by colectomy and ileorectal anastomosis remained constant over the first two periods but fell considerably in the last. The number of patients treated by total proctocolectomy remained fairly constant throughout but fell dramatically when considered as a percentage of the operations performed. Two patients had a Kock intra-abdominal pouch fashioned simultaneously with their total proctocolectomy and three had one at a later stage.

There were two postoperative deaths both resulting from pulmonary embolus after further surgery among patients originally treated electively. One was after repair of a paraileostomy hernia after a total proctocolectomy, and the other after laparotomy for drainage of intra-abdominal sepsis after restorative proctocolectomy.

\section{URGENT SURGERY}

One hundred and six patients were operated upon urgently and the operations carried out were total proctocolectomy 12 , conservative proctocolectomy two, colectomy and ileostomy (with rectal preservation) 86, colectomy and ileorectal anastomosis four, and restorative proctocolectomy one. One further patient initially thought to have Crohn's colitis had a two stage colectomy. There were three postoperative deaths; in 1980, 1981, and 1990. One was due to bronchopneumonia in a man of 76 after a colectomy and ileostomy and the second to uncontrollable haemorrhage from a duodenal ulcer that had eroded the gastroduodenal artery. The third death occurred in 1990 in a lady of 82 . It had been hoped to avoid surgery in this patient but the colitis failed to respond to medical treatment. The patient was treated by a colectomy and ileostomy but never progressed satisfactorily postoperatively. She could not come to terms with the ileostomy, refused to eat and drink, and finally died of bronchopneumonia some six weeks after surgery.

\section{Subsequent outcome}

\section{COLECTOMY AND ILEOSTOMY: ELECTIVE}

SURGERY

Thirty four patients had a colectomy and ileostomy performed as the initial elective treatment of colitis. By the end of 1990, 10 patients had had the rectum excised, in one an ileorectal anastomosis had been fashioned, in 13 the rectum had been removed and a pelvic pouch constructed: in eight the decision was still pending and two in whom the operation was a palliative procedure had died of carcinomatosis. In the third five year period 1986-90 almost half of the patients ( 8 of 17) had a pelvic pouch as the second stage procedure and only one had the rectum excised.

\section{URGENT SURGERY}

Of the 85 survivors of urgent operations (84 after colectomy and ileostomy and one after a left hemicolectomy then right hemicolectomy) with the rectum left, 35 came to rectal excision (conservative in one); 35 had restorative 

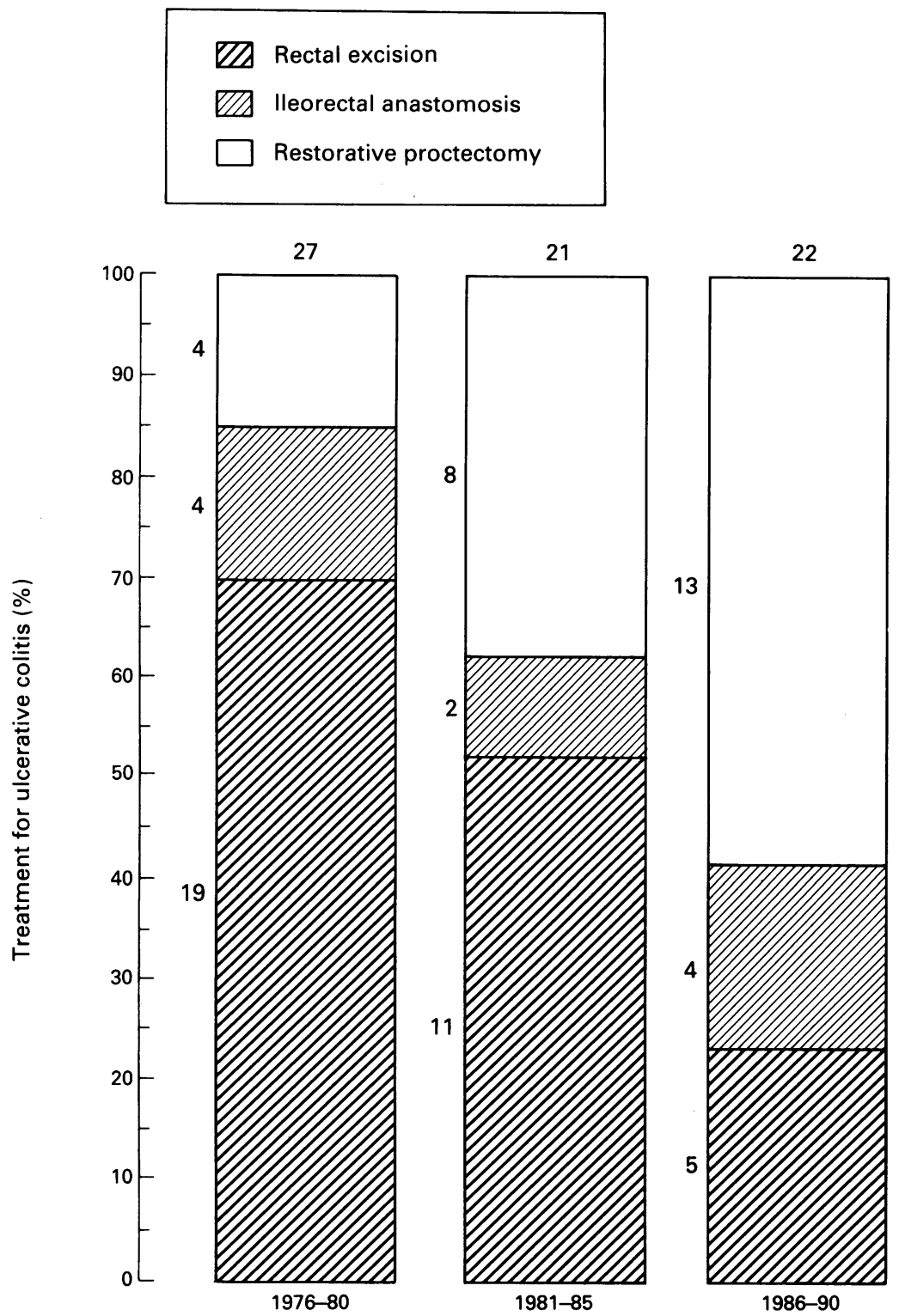

Figure 2: Fate of the rectum in 70 patients having colectomy and ileostomy as initial urgent treatment for ulcerative colitis.

operations (ileorectal anastomosis 10, ileoanal pouch 25), in 13 (mainly those operated on recently) a decision was still pending at the end of 1990, and two elderly patients had died of unrelated causes. If the last 15 patients are excluded, then the number and percentage of patients having one of these three options are shown in Figure 2 where it can be seen that the progressive fall in rectal excision over the three five year periods is matched by the rise in those coming to restorative proctectomy while the percentage having an ileorectal anastomosis has remained fairly constant. The Table shows the fate of the rectum in all 119 operation survivors.

Fate of the rectum in 119 patients after colectomy and ileostomy as the initial treatment

\begin{tabular}{lllllcl}
\hline $\begin{array}{l}\text { First } \\
\text { operation }\end{array}$ & Number & $\begin{array}{l}\text { Rectal } \\
\text { excision }\end{array}$ & $\begin{array}{l}\text { Ileorectal } \\
\text { anastomosis }\end{array}$ & $\begin{array}{l}\text { Restorative } \\
\text { proctectomy }\end{array}$ & $\begin{array}{l}\text { Decision } \\
\text { pending }\end{array}$ & Dead \\
\hline Elective & 34 & 10 & 1 & 13 & 8 & 2 \\
Urgent & 85 & $35^{\star}$ & 19 & 25 & 13 & 2 \\
\hline
\end{tabular}

^Conservative in one.
ILEORECTAL ANASTOMOSIS

By the end of 1990, of the 61 patients who had this operation as primary elective treatment the ileorectal anastomosis was no longer functional in $11(18 \%)$ patients. One patient with cirrhosis and gross ascites postoperatively was treated by a loop ileostomy, which has not been closed. Four patients with intractable diarrhoea were treated by rectal excision (leaving the anal sphincters in two). In six patients the rectum has been removed (for dysplasia in one and diarrhoea in five) and an ileoanal pouch constructed with a successful outcome in the four patients so treated at this hospital.

Four patients were treated urgently by this operation: the anastomosis remains functional in each case.

For the 11 patients who had an ileorectal anastomosis as secondary treatment, this failed in the one patient so treated after an elective colectomy and ileostomy but was successful in all 10 after an urgent colectomy and ileostomy.

Thus the overall success rate of ileorectal anastomosis was $84 \%$; 64 of 76 patients.

\section{PELVIC POUCH}

Of 115 patients treated by restorative proctocolectomy (elective 114, urgent one), the operation has been unsuccessful in six $(5 \%)$ : failure is defined as a non-functioning pouch (removal or longterm ileostomy). In one patient, ischaemia of the small bowel necessitated the removal of the pouch in the postoperative period. Bowel frequency in two, pouchitis in one, an anal fissure and sepsis in one, and a pouch-vaginal fistula in one have been responsible for failure in the other five.

One of the 13 patients with a pouch constructed after elective colectomy and ileostomy needed conversion to a standard ileostomy because of diarrhoea and incontinence.

Of the 25 patients who had a pouch after an urgent colectomy and ileostomy, this has been a failure in four leading to formation of an end ileostomy by the end of 1990 (diarrhoea/incontinence three, sepsis one).

Pouch success has been defined as a functional ileoanal reservoir at the end of 1990 . Overall, therefore, the success rate was $93 \%$ : 142 of 153 patients still had a functional reservoir. Two patients have been converted to a Kock abdominal pouch: the remainder have an end ileostomy.

Of the total of five patients with Kock abdominal pouch, this has been a success in three patients.

\section{CONSERVATIVE PROCTOCOLECTOMY}

Conservative proctocolectomy undertaken with the twin aims of avoiding a troublesome perineal wound and retaining the option of a pelvic pouch in the future has not been entirely successful in either. None of the 12 patients so treated electively has been converted to a pouch although two patients offered the option have refused it. Pelvic sepsis has led to excision of the anus in three and division of the 
sphincters in one. Of the remaining patients, most complain of some anal discharge.

Of the two patients treated urgently by conservative proctocolectomy, one had a pouch constructed after six months but the other required excision of the anal stump two years later.

\section{Discussion}

\section{ELECTIVE SURGERY}

There is no perfect operation for ulcerative colitis. Elderly patients may be unsuitable for restorative procedures and patients with a low rectal malignancy require total proctocolectomy. Some younger patients after full explanation and meeting with an ileostomist choose this procedure as the one most likely to be trouble free and with only one operation needed. Over a third of these patients, however, will require readmission for small bowel obstruction or other sequelae of this operation. ${ }^{4}$ One of the late deaths in this series was in a patient after operation for a parastomal hernia after a total proctocolectomy.

Colectomy and ileorectal anastomosis is possible if the rectum has sufficient compliance to minimise stool frequency and reduce the chance of urge incontinence. Where chronic rectal disease has led to contraction with a reduction in capacity, ileorectal anastomosis is contraindicated. The operation is associated with a short hospital stay, a low morbidity and, in this series, a success rate of $84 \%$. Annual surveillance is essential afterwards because of the risk of malignancy. ${ }^{5}$ Nowadays this operation is a more attractive option as failure (functional disturbance including diarrhoea and urgency resulting from persisting inflammation) no longer means rectal excision as almost all such patients are suitable for restorative proctectomy.

When a restorative proctocolectomy is advised the question arises whether it should be done as a single procedure or preceded by a colectomy and ileostomy with preservation of the rectal stump. The reason for colectomy and ileostomy as a first stage elective operation was not always apparent from a study of the case notes. The most commonly stated was that the patient was not considered fit enough for a one stage procedure. Another was that at the time the most appropriate procedure could not be decided either by the surgeon or, perhaps more importantly, by the patient. A staged procedure thus gave the opportunity for a definitive decision to be made later.

A further decision has to be made regarding the use of a defunctioning ileostomy. While there is a trend at present to try and perform pouch surgery without a covering loop ileostomy ${ }^{67}$ most restorative proctocolectomies described here were defunctioned. In assessing the results of pouch surgery described here, it must be remembered that the pouches performed were constructed in a variety of fashions ( $S$ pouch, $J$ pouch, $\mathrm{W}$ pouch, and Kock pouch placed in the pelvis). Many of the operations were performed early in the development of this operation and it is hoped that the results achieved now would be better. The longterm results of this operation have yet to be assessed, but the initial success rate of 142 of 153 is encouraging.

\section{URGENT SURGERY}

The first priority for patients with acute colitis is the preservation of life. The safest and simplest operation is a colectomy and ileostomy with the rectum preserved as a mucous fistula. This operation has the advantage of preserving the anal sphincters and of making subsequent formation of a pelvic reservoir easier. In this series no patient so treated required urgent excision of the rectum in the first weeks after this procedure.

Although 12 patients were treated by total proctocolectomy as the initial procedure, the reasons for this in the urgent situation were not always obvious. The operations were fairly evenly spread over the 15 years and therefore did not reflect earlier thinking that a total proctocolectomy was the ideal operation. Most of these patients although classified as needing urgent surgery (by very strict criteria) were not so severely ill that a one stage total proctocolectomy was out of the question. In two, anal lesions suggested the possibility of Crohn's disease, in two severe left sided disease, and bleeding in another two were all factors that might have led to the decision. In one instance, the operation was at the patient's request.

Five patients were treated urgently by restorative procedures; four by colectomy and ileorectal anastomosis and one by restorative proctocolectomy. Paradoxically this was the first restorative proctocolectomy performed at this hospital and, although the patient had a satisfactory outcome, it was immediately felt that this was a procedure that should be reserved for the elective case. The reason for avoiding restorative procedures in acute disease is the risk of anastomotic breakdown and haemorrhage. Anastomotic breakdown is presumed to be more likely because acutely ill patients are usually malnourished, toxic, and receiving high doses of corticosteroids. Haemorrhage is increased when the pelvis is dissected in the presence of severe rectal disease and although Lee and Truelove ${ }^{8}$ have previously shown that this problem can be overcome, we have endeavoured to minimise morbidity of the pouch procedure.

If the rectum is to be preserved in the acute situation, how much should be retained? The answer is, as much as possible. This is to facilitate a subsequent restorative procedure. Where a long rectal or rectosigmoid stump has been left, it is easy to find at a later operation and performing a close rectal dissection is straightforward. If the rectum has been partially removed, the dissection required during a subsequent restorative proctectomy may be hazardous with the risk of nerve injury and damage to the vagina and the base of the bladder and vesicles in men. 
Where a long stump has been preserved this has usually been in the form of a mucous fistula brought out through the bottom of the abdominal wound. This has the disadvantage that some patients develop a troublesome discharge from the fistula. Alternative procedures include intraperitoneal closure of the stump by hand suture or stapling device. Alternatively the end of the distal sigmoid can be brought up to the anterior rectus sheath closing the skin over the top of it. The feasibility of closing the rectosigmoid stump depends on the severity of the distal disease and may not be possible. While most cases have been treated by exteriorisation in this series, it is possible that with careful selection more patients might have avoided a mucous fistula.

Some patients having had an urgent colectomy and ileostomy may be quite happy with their ileostomy and decide that they do not want a restorative procedure. The problem is to decide in such a case if the risks of subsequently removing the rectum are greater than the risks of leaving it behind. Retaining the rectum in such a case carries the risk of a troublesome rectal discharge, rectal bleeding, and a risk of carcinoma. ${ }^{9}$ It is sometimes difficult to examine the rectum in such patients because it becomes narrowed and atrophic and examination is often painful. Clearly patient preference plays a part but the advice to younger patients who do not want a restorative procedure should initially be to wait and see. When they are sure they wish to retain the ileostomy and lose the rectum, proctectomy using an intersphincteric dissection is generally advised. ${ }^{10}$

Where patients choose to have a restorative procedure, conversion to an ileorectal anastomosis is often not possible because of persisting rectal inflammation causing shrinkage because of fibrosis. In the 10 patients in this study who did have such a conversion, the result was satisfactory in each case. In these it must be presumed that the rectum was judged by the clinician to be in a sufficiently good state to act as an adequate reservoir. In those patients who have had restorative proctectomy, a successful outcome was achieved in 21 of 25 cases selected for this procedure.

It is obvious from the figures presented here that the trend in recent years at this hospital has been towards restorative surgery whenever possible. Even allowing for some bias in the referral pattern for elective surgery, the rise in restorative proctocolectomy from $7 \%$ in $1976-80$ to $52 \%$ in $1986-90$ is striking. For patients having a two stage procedure the swing away from rectal excision towards restorative proctectomy is equally noticeable. In the years $1976-80,69 \%$ came to rectal excision and $17 \%$ had a pelvic pouch: by 1986-90 the reverse was true: in $19 \%$ the rectum had been excised and $68 \%$ went on to a pouch. At the Mayo Clinic between 1976 and 1986, just under a third of the patients had a total proctocolectomy and just under half a restorative proctocolectomy. ${ }^{11}$ These figures are quite similar to those presented here. Their data differ from ours in the much higher proportion having a Kock ileostomy (18\%) and much lower proportion treated by colectomy and ileorectal anastomosis $(3 \%)$.

Not all patients are suitable for a pelvic pouch and some still opt for a total proctocolectomy after a full explanation and meetings with patients in both categories. Colectomy and ileorectal anastomosis remains an attractive option in suitable patients: after an urgent colectomy and ileostomy it has been particularly successful. The enormous recent interest in restorative proctocolectomy should not be allowed to obscure the fact that other operations for ulcerative colitis still have their place.

The results of surgery for ulcerative colitis performed at St Mark's Hospital between 1976 and 1990 have been analysed to assess changing patterns in surgery over this period and the outcome of the various procedures. Only patients who had their initial surgery at St Mark's have been included: there were 316 patients who had elective surgery and 106 who had urgent operations. There were three deaths related to elective surgery and three deaths related to urgent surgery. Among patients undergoing elective operations, there has been a shift away from total proctocolectomy $(60 \%$ of cases $1976-80 ; 21 \%$ of patients 1986-90). The overall success rate - that is, a still functional anastomosis - for colectomy and ileorectal anastomosis was $84 \%$ (64 of 76 patients) and for restorative proctocolectomy was $93 \%$ (142 of 153 patients).

1 Parks AG, Nicholls RJ. Proctocolectomy without ileostomy for ulcerative colitis. $B M 7$ 1978; 2: 85-8.

2 Setti-Carraro P, Ritchie JK, Wilkinson $\mathrm{KH}$, Nicholls RJ, Hawley PR. The first 10 years' experience of restorative proctocolectomy for ulcerative colitis. Gut 1994; 35: proctocolec $1070-5$.

3 Ewart WB, Lennard-Jones JE. Corticosteroids in preoperative medical management of ulcerative colitis. Do they affect surgical success? Lancet 1960; ii: $60-4$

4 Phillips RKS, Ritchie JK, Hawley PR. Proctocolectomy and ileostomy for ulcerative colitis; the longer term story. fR Soc Med 1989; 82: 386-7.

5 Baker WNW, Glass RE, Ritchie JK, Aylett SO. Cancer of the rectum following colectomy and ileorectal anastomosis for ulcerative colitis. Br $\mathcal{F}$ Surg 1978; 65: 862-8.

6 Everett WG, Pollard SG. Restorative proctocolectomy without temporary ileostomy. Br f Surg 1990; 77: 621-2.

7 Sager PM, Lewis W, Holdsworth PJ, Johnston D. One-stage restorative proctocolectomy without temporary ileostomy. Dis Colon Rectum 1992; 35: 582-8.

8 Dis Colon Rectum 1992; 35: 582-8. colitis. World f Surg 1980; 4: 195-201.

9 Ritchie JK. The causes of late mortality in ileostomists. Proc $R$ Soc Med 1972; 65: 73 .

10 Leicester RJ, Ritchie JK, Wadsworth J, Thomson JPS Hawley PR. Sexual function and perineal wound healing after intersphincteric excision of the rectum for inflammatory bowel disease. Dis Colon Rectum 1984; 27: 244-8.

11 Goudet P, Dozois RR, Kelly KA, Melton LJ, Ilstrup DM Phillips SF. Changing referral patterns for surgery of chronic ulcerative colitis. Ann Chir 1992; 46: 9-10. 